\title{
Inversión Extranjera Directa y Crecimiento Económico en Latinoamérica
}

\author{
Agustín Álvarez Herranz ${ }^{1}$, J. Santiago E. Barraza ${ }^{2}$ y Ana M. Legato ${ }^{2}$ \\ (1) Universidad de Castilla-La Mancha, Facultad de Ciencias Económicas y Empresariales, \\ Pza. de la Universidad 1, E-02071 Albacete-España (e-mail: Agustin.Alvarez@uclm.es) \\ (2) Universidad Nacional del Centro de la Provincia de Buenos Aires, Facultad de Ciencias \\ Económicas, Centro de Estudios en Administración (CEA), Pinto 399, (7000) Tandil, Buenos \\ Aires-Argentina (e-mail: barraza@econ.unicen.edu.ar; legato@econ.unicen.edu.ar)
}

\begin{abstract}
Resumen
En este trabajo se estudia el impacto de la Inversión Extranjera Directa en el Crecimiento Económico en 14 países latinoamericanos. Para ello, se analizó su relación con el incremento del Producto Interno Bruto per cápita (como aproximación al Crecimiento Económico) y con la acumulación de Capital Privado per cápita durante el período comprendido entre 1996 y 2003. A tal efecto, se desarrolló un modelo de dos ecuaciones simultáneas que son resueltas utilizando análisis de datos panel de efectos fijos, tanto para el análisis de países como para estudiar el efecto en el transcurso del tiempo. Los resultados hallados permiten concluir que, en los países Latinoamericanos estudiados, la Inversión Extranjera Directa impulsa tanto la acumulación de Capital Privado per cápita como el incremento del Ingreso per cápita. La Calidad de las Instituciones, y principalmente la protección de la propiedad privada y la libertad de los individuos, es determinante para el Crecimiento. Se deduce también, que la Inversión Pública también impulsa el Crecimiento y que los países considerados tienden a converger condicionalmente, concordante con información de la literatura.
\end{abstract}

Palabras clave: inversión extranjera directa, crecimiento, inversión privada, Latinoamérica, datos panel

\section{Foreign Direct Investment and Growth in Latin America}

\begin{abstract}
In this paper a study on the impact of Foreign Direct Investment on Economic Growth in 14 Latin American countries is presented. With this purpose the relationship of Foreign Direct Investment per capita with Gross Domestic Production per capita variation (as a proxy for Economic Growth) and Private Capital per capita variation during the period 1996-2003 were analyzed. A model with two simultaneous equations that are solved using fixed-effects panel for both analysis of countries and the study of the time effect. The results allows concluding that for those countries studied the Foreign Direct Investment boosts both the Private Capital per capita accumulation and the Income per capita growth. The Institutional Quality, mainly that related to private property rights protection and individual freedom, are determining factors for Growth. It was finally found that Public Investment also boosts Growth and the countries considered in the analysis show conditional convergence, which is consistent with the mainstream literature.
\end{abstract}

Keywords: foreign direct investment, growth, private investment, Latin America, panel data 


\section{INTRODUCCIÓN}

El grado de éxito de las políticas económicas se mide, típicamente, por su impacto en el Crecimiento Económico de los países. Al tiempo que la movilidad de capitales ha aumentado de manera importante, en las últimas décadas este factor se ha convertido en objeto típico de estudio por su posible impacto en el Crecimiento Económico, sea desde la perspectiva de la convergencia como de la difusión de la tecnología.

Los modelos económicos asignan diferentes roles a la Inversión Extranjera Directa en la explicación del Crecimiento Económico de acuerdo a los supuestos de los cuales partan. En términos generales, los modelos neoclásicos asumen que la Inversión Extranjera Directa no impacta en el Crecimiento Económico en el largo plazo, mientras que los modelos endógenos asumen que sí lo hace.

En general, ha sido demostrado que los niveles de Movilidad del Capital afectan el crecimiento de los países, y se ha observado que el fenómeno de la Globalización ha potenciado tal movilidad. La característica fundamental que diferencia a la Inversión Extranjera Directa, estudiada en este trabajo, de las Inversiones Extranjeras Financieras es su permanencia en la economía destinataria. En otras palabras, la Inversión Extranjera Directa es menos volátil que otros tipos de inversiones.

El presente trabajo estudia el papel de la Inversión Directa Extranjera per cápita, como variable representativa de la movilidad de capital, en el Crecimiento y Convergencia de los países latinoamericanos en el período 1996-2003. Con este fin, se desarrolla un modelo multiecuacional, con datos de panel de 14 países latinoamericanos, y se pretende corroborar la existencia de una relación positiva entre Inversión Directa Extranjera per cápita y Crecimiento Económico, condicionada al comportamiento de otras variables explicativas según propuesto por Borensztein et al. (1995); Hermes y Lensink (2000); Alfaro et al. (2004), entre otros. En nuestro caso, las variables controladas representan el nivel de Capital Humano, la interacción entre Capital Humano e Inversión Extranjera Directa, la Calidad Institucional, los niveles de Capital Público y Privado per cápita y los niveles previos de Ingreso per cápita.

En línea con Barro y Sala-i-Martin (1990) y Blomström et al. (1994), se asume que los países convergen en términos de sus Ingresos per cápita, esto condicionado a que se controlen otras variables, como antes mencionado.

Dado que, si se asumen oportunidades de inversión acotadas en una economía, podría esperarse que los inversores extranjeros compitieran con los domésticos por tales oportunidades, entonces podría esperarse también que la Inversión Extranjera Directa desplazase, al menos parcialmente, a la Inversión Doméstica. Cabe entonces preguntarse cual es el impacto de la Inversión Extranjera Directa en la acumulación de Capital Privado per cápita, como lo hacen Borensztein et al. (1995). Por este motivo, incluimos en nuestro modelo una ecuación en la que el Capital Privado per cápita es función de la Inversión Extranjera Directa per cápita, además de la interacción entre Capital Humano e Inversión Extranjera Directa y variables proxy para la Calidad Institucional de los países.

La selección de la muestra corresponde al interés por estudiar el impacto de la Inversión Extranjera Directa en las economías latinoamericanas, aplicando una metodología innovadora en cuanto a análisis de datos longitudinales en un modelo modelo multiecuacional y esto constituye, a juicio de los autores de este trabajo, el aporte más importante del trabajo a la literatura.

Los hallazgos del trabajo coinciden con lo señalado por gran parte de la literatura reciente, revisado y compilado por Ozturk (2007). Esto es, la Inversión Extranjera Directa tiene un efecto positivo y significativo tanto en la Formación de Capital como en el Crecimiento Económico.

Parte de la literatura señala la importancia de los diferentes sectores de la economía en la explicación del fenómeno de convergencia. Si bien se considera importante analizar esta alternativa, no lo se ha realizado en el presente trabajo por restricciones de datos y que sin embargo, es un área para realizar investigaciones futuras. 


\section{MARCO TEÓRICO}

La relación teórica entre Inversión Extranjera Directa y Crecimiento Económico difiere de acuerdo al marco de análisis utilizado. Los modelos de crecimiento neoclásicos implican que la Inversión Extranjera Directa no afecta al Crecimiento Económico en el largo plazo, como resultado de suponer mercados de competencia perfecta, productividad marginal decreciente y retornos constantes a escala. A partir de estos supuestos, los incrementos exógenos de la Inversión Extranjera Directa sólo pueden afectar positivamente el capital por persona transitoriamente, dados los retornos decrecientes. De este modo, la única manera de afectar el Crecimiento Económico en el largo plazo es a través de modificar dos factores exógenos: la tecnología y el trabajo.

En contraste, los modelos de crecimiento endógeno indican, en general, que la Inversión Extranjera Directa tiene un efecto positivo en el Crecimiento Económico de manera indirecta a través tanto de la Formación de Capital como del desarrollo de los recursos humanos.

En términos generales, la literatura señala que los efectos de la Inversión Extranjera Directa se producen a través de las externalidades que produce, como la transferencia de tecnología y los spillovers. Romer (1993), afirma que la Inversión Extranjera Directa puede facilitar la transferencia de tecnología y know-how desde los países más desarrollados hacia los menos desarrollados, incrementando la productividad de todas las firmas, dada la existencia de spillovers que benefician a la economía en su conjunto. Borensztein et al. (1995), señalan que la Inversión Extranjera Directa constituye un vehículo para la transferencia de tecnología y que contribuye al crecimiento en mayor medida que la Inversión Interna. Sin embargo, observan que dichos resultados están condicionados a la existencia de ciertos factores en el país de destino, como un nivel mínimo dado en el capital humano.

Alfaro et al. (2004), coinciden en el condicionamiento de los efectos de la Inversión Extranjera Directa, esta vez apuntando al desarrollo de los mercados financieros como el factor clave. Hermes y Lensink (2000), señalan que tanto el grado de desarrollo de los mercados financieros como el nivel capital humano existente condicionan el impacto positivo. Balasubramanyam et al. (1996), focalizan el grado de apertura de la economía. Blomström et al. (1994), apuntan al nivel de ingresos y afirman que aquellos países en desarrollo con mayores niveles de ingresos obtendrán los mayores beneficios.

En contraste, estudios tales como los de Boyd y Smith (1992), afirman que, dada la existencia de distorsiones financieras y comerciales, la Inversión Extranjera Directa produce efectos negativos sobre el Crecimiento Económico. En el medio entre estos resultados dispares, existen trabajos como De Mello (1999); Lipsey (2000); Carkovic y Levine (2005), entre otros, donde no se logra establecer una relación positiva significativa entre Inversión Extranjera Directa y Crecimiento Económico.

En los análisis siguientes se considera también el proceso de convergencia real entre los países. En este sentido, la literatura, en gran medida construida a partir de Barro y Sala-i-Martin (1990), señala que existe convergencia condicionada de economías y que el capital tiende a desplazarse desde aquellos países con bajos retornos marginales -i.e. países desarrollados- hacia países con tasas más altas -i.e. países menos desarrollados-.

Galindo y Escot (2004), prueban que los países menos desarrollados crecen más rápidamente que aquellos desarrollados, siempre y cuando los niveles de liquidez y riesgo de los países analizados sean similares. Blomström et al. ( 1994), confirman la existencia de convergencia condicionada a los niveles de educación, los cambios en la tasa de participación de la fuerza laboral, la inversión extranjera, las estructuras de precios y la tasa de inversión fija.

\section{METODOLOGÍA}

Selección de la muestra

La muestra analizada abarca datos del período 1996-2003 para los países: Argentina, Bolivia, Brasil, Colombia, Costa Rica, República Dominicana, Ecuador, Salvador, Guatemala, México, Nicaragua, 
Paraguay, Perú y Uruguay. La selección de los mismos se corresponde con el interés de este trabajo, por estudiar el impacto de la Inversión Extranjera Directa en las economías latinoamericanas aplicando una metodología innovadora en cuanto a análisis de datos longitudinales en un modelo modelo multiecuacional y la disponibilidad de información homogénea para el período de análisis.

En lo referente a las variables explicativas del presente trabajo consideradas como determinantes del crecimiento económico y de la acumulación de capital privado, estas surgen del aporte de la literatura especializada, como mencionado anteriormente, y son las siguientes:

- $\quad Y_{i t}=$ Producto Interno Bruto per cápita en billones de dólares, a valores de paridad del poder adquisitivo (PPS) internacionales constantes 2000.

- $\operatorname{PrK}_{\text {it }}=$ Capital Privado per cápita en billones de dólares, a valores de paridad del poder adquisitivo (PPS) internacionales constantes 2000. La variable se ha calculados a partir de los datos de los porcentajes de participación en el PIB.

- $\quad \mathrm{PuK}_{\mathrm{it}}=$ Capital Público per cápita en billones de dólares, a valores de paridad del poder adquisitivo (PPS) internacionales constantes 2000. La variable se ha calculados a partir de los datos de los porcentajes de participación en el PIB.

- $\mathrm{GOB}_{\mathrm{it}-1}=$ Índice de Gobernabilidad, incluida con un retardo de un período. Mide el ejercicio del poder público con el objeto de obtener ganancias privadas. Es una variable no observable que resulta de aplicar el método de componentes principales a las cuatro dimensiones de la Calidad Institucional que brinda el Banco Mundial (Kaufman et al., 2005): (1). Voz y Representación, que mide los derechos políticos, civiles y humanos; (2) Eficiencia de Gobierno, que mide la competencia de la burocracia; (3) Imperio de la Ley, que mide la calidad de las instituciones para hacer cumplir los contratos, el poder de policía y el sistema judicial; (4) Control de Corrupción.

- $\quad \mathrm{KHU}_{\mathrm{it}}=$ Capital Humano. Es aproximado a través de la variable Gasto Público en Educación per cápita en billones de dólares, a valores de paridad del poder adquisitivo (PPS) internacionales constantes 2000.

- $\quad \mathrm{IED}_{\text {it }}=$ Inversión Extranjera Directa per cápita en billones de dólares, a valores de paridad del poder adquisitivo (PPS) internacionales constantes 2000.

- $\quad \mathrm{IED}_{\mathrm{it}}{ }^{*} \mathrm{KHU}_{\mathrm{it}}=$ Variable creada con el propósito de medir el efecto combinado de la Inversión Extranjera Directa per cápita y el Capital Humano per cápita. Esta expresada en billones de dólares, a valores de paridad del poder adquisitivo (PPS) internacionales constantes 2000. Cuando aparece expresada en el periodo "t-1" es para evitar la multicolinealidad con la variable $\mathrm{KHU}_{\text {it }} \mathrm{y}$ la variable IED it.

- $\mathrm{DP}_{\mathrm{it}-1}=$ Derechos Políticos (DP) es el rating por países de Freedom in The World. Se utiliza una escala de 1 a 7 para indicar el grado de libertad reinante en cada país, con 1 representando el máximo grado de libertad y 7 el más bajo.

El subíndice "i" indica el i-ésimo país y el subíndice "t", el momento de tiempo. La fuente para todas las variables es el Banco Mundial, con excepción de DP, obtenida de Freedom in The World.

\section{El modelo}

El modelo econométrico desarrollado en este trabajo tiene un doble objetivo: por un lado medir el impacto de la Inversión Extranjera Directa en el Crecimiento Económico de los países latinoamericanos y, por el otro, determinar cómo influye en la Formación de Capital. El modelo especificado incluye dos ecuaciones simultáneas que cubren estos objetivos.

La elección de un modelo multi-ecuacional se debe al reconocimiento de las limitaciones de los modelos uni-ecuacionales, los cuales recogen solamente la relación existente entre una variable 
endógena $(\mathrm{Y})$ y un conjunto de variables exógenas (Xi) con lo cual basta con conocer el comportamiento de las variables explicativas para realizar estimaciones acerca de la variable endógena. Esta especificación no es la más adecuada para estudiar la interdependencia de las variables económicas, entre las que la relación no siempre es unidireccional.

Los modelos de ecuaciones simultáneas, en contraste, permiten modelizar estructuras relacionales más complejas para las variables, y pueden recoger no sólo la influencia de la variable endógena sobre las predeterminadas, sino también las relaciones que entre éstas puedan existir.

Se parte por reconocer que los estudios empíricos de Crecimiento Económico generalmente se basan en una ecuación que es una variante del modelo de Cobb-Douglas, y que adopta la siguiente especificación:

$\ln ($ Yit $)-\ln ($ Yit- $\pi)=k+\beta \ln ($ Yit- $\pi)+\delta$ Wit- $\pi+\eta i+\zeta i+\varepsilon i t$

Donde, para el presente estudio, Yit es el PIB per-cápita, en el país i en el período t, Wit- $\tau$ es un vector fila de determinantes, es decir, de variables explicativas de Crecimiento Económico, $\eta$ i es el componente fijo y específico de cada país, $\zeta$ t es una constante específica de cada período y cit es el término de error. Esta es una expresión general de una ecuación de crecimiento económico con convergencia. El vector fila de determinantes, o variables explicativas del modelo, Wit- $\tau$ incluye en nuestro caso a PrK que representa el Capital Privado per cápita, PuK por Capital Público per cápita, GOB por el Índice de Gobernabilidad, KHU por el Capital Humano per cápita, IED representando la Inversión Extranjera Directa per cápita e IED*KHU es el efecto combinado de la Inversión Extranjera Directa per cápita y el Capital Humano per cápita.

Las ecuaciones de este tipo, utilizadas en estimaciones de corte transversal derivan de los modelos empleados por Barro y Sala-i-Martin (1990), en los que prueban la existencia de convergencia condicional para los estados de USA a una tasa de alrededor de $2,5 \%$ en el período comprendido entre 1840 y 1988. También encuentran evidencia de convergencia condicional a una tasa de 2,0\% anual para una muestra de 98 países en el período comprendido entre 1960 y 1985.

El método de estimación a emplear es el de Mínimos Cuadrados Generalizados (EGLS) mediante el método robusto de White para heteroscedasticidad entre secciones cruzadas, para luego aplicar el Método de Mínimos Cuadrados en dos etapas (Two-Stage EGLS ), también mediante el método robusto de White para heteroscedasticidad entre secciones cruzadas.

La elección de la metodología de estimación se debe a que los Mínimos Cuadrados Ordinarios no tienen en cuenta el efecto individual no observable, el cual debiera ser no-correlacionado con el resto de las variables explicativas, algo que no se cumple. A su vez, existe también un problema relacionado con la endogeneidad de los regresores empleados. Caselli et al. (1996), introducen una nueva metodología al estudio del Crecimiento Económico y la convergencia, la cual consiste en la estimación de paneles dinámicos y corrige los problemas de endogeneidad, sesgo por variable omitida e inconsistencia. Con esto inducen un salto en la estimación del coeficiente de convergencia, sobre la misma muestra, del 2 al $10 \%$ anual aproximadamente, lo cual implica que las economías están permanentemente cerca de un estado estacionario. En la estimación del modelo de Solow simple y en su versión aumentada, el test de Hausman rechaza la exogeneidad estricta de cada conjunto de variables para ambos modelos. Por lo tanto, se obtienen parámetros consistentes. Bond et al. (2001), describen la forma general de la aproximación hecha por Caselli et al. (1996), escribiendo la ecuación a regresionar como un modelo de paneles de datos dinámicos, tomando las primeras diferencias y removiendo el efecto específico de cada país no observable e invariable al tiempo. Emplean como variables explicativas series rezagadas dos períodos o más en niveles, bajo el supuesto de que las variaciones temporales de los errores en la ecuación original en niveles no están serialmente correlacionadas. Esto permite obtener estimadores consistentes, que demuestran que los resultados del coeficiente de convergencia son bastante menores que los hallados por Caselli et al. (1996), dado que se solucionan los problemas de sesgo del estimador de primera etapa. 


\section{RESULTADOS Y DISCUSIÓN}

En primer lugar se realiza un análisis empírico considerando tanto los efectos directos como los efectos indirectos de la Inversión Extranjera Directa per cápita sobre el Producto Interno Bruto per cápita. Para analizar los efectos directos, se incluye la Inversión Extranjera Directa per cápita en la ecuación de determinación del Producto Interno Bruto (PIB) per cápita mientras que para analizar los indirectos, se la incluye en la ecuación de determinación de la Inversión Privada per cápita.

Según lo especificado en la ecuación (1), esta representa un modelo de efectos fijos, lo cual permite incorporar las diferencias entre los países basándose en la siguiente ecuación de convergencia:

$\Delta \ln \left(y_{i t}\right)=\alpha_{i}+\beta \ln \left(y_{i t-1}\right)+\delta_{j} W_{j i t-h}+\varepsilon_{i t}$

Donde $y_{i t}$ es el PIB per cápita del i-ésimo país en el momento $t ; \alpha_{i}$ son los efectos individuales de cada uno de los i-ésimos países y tiempo $t ; \beta$ es el parámetro de convergencia; $W_{\text {jith }}$ es un vector fila de determinantes del Crecimiento Económico; $\varepsilon_{i t}$ es el término de error.

A partir de las expresiones generales (1) y (2), las ecuaciones a estimar adoptan la siguiente forma:

$\mathrm{d} \ln (Y)_{\mathrm{it}}=\beta_{0}+\beta_{1} \ln (\mathrm{Y})_{\mathrm{it}-1}+\beta_{2} d \ln (\operatorname{PrK})_{\mathrm{it}}+\beta_{3} \mathrm{~d} \ln (\mathrm{PuK})_{\mathrm{it}}+\beta_{4} \mathrm{GOB}_{\mathrm{it}-1}+\beta_{5} \mathrm{~d} \ln (\mathrm{KHU})_{\mathrm{it}}+\beta_{6} \mathrm{~d} \ln (\mathrm{IED})_{\mathrm{it}}+$ $\beta_{7} d \ln (\text { IED * KHU })_{\mathrm{it}-1}+\varepsilon_{\mathrm{it}}$

$\ln (\operatorname{PrK})_{\mathrm{it}}=\beta_{8}+\beta_{9} \ln (\mathrm{IED})_{\mathrm{it}-1}+\beta_{10} \ln \left(\mathrm{IED}{ }^{*} \mathrm{KHU}\right)_{\mathrm{it}}+\beta_{11}(\mathrm{GOB})_{\mathrm{it}}+\beta_{12}(\mathrm{DP})_{\mathrm{it}-1}+\varepsilon_{\mathrm{it}}$

La ecuación (3) corresponde al incremento Producto Interno Bruto per cápita, donde las variables corresponden a las definiciones hechas en la sección Metodología.

En el análisis ex ante se esperan signos positivos para la Inversión Privada per cápita, el Capital Humano per cápita, el Índice de Gobernabilidad, la Inversión Extranjera Directa per cápita y la combinación de Inversión Extranjera Directa per cápita y Capital Humano per cápita, de acuerdo a lo enunciado por gran parte de la literatura existente y previamente mencionado. Para el PIB per cápita se espera un signo negativo, dado que se asume un proceso de convergencia.

El signo esperado para el Capital Público per cápita no es claro ex ante. Por un lado, existen estudios como Bertola (1993); Alesina y Rodrik (1994); Persson y Tabellini (1994), entre otros, que muestran que las Políticas Públicas Activas tienden a desplazar a la Inversión Privada e impactan negativamente en el Crecimiento Económico. Por otro lado, autores como Bénabou (1996a; 1996b) y Bourguignon y Verdier (2000), afirman que las políticas redistributivas que implican Inversión Pública tienen un impacto positivo en el Crecimiento Económico si contribuyen a reducir imperfecciones de los Mercados de Créditos o las restricciones de liquidez que afectan negativamente tanto a la inversión en capital físico como humano (Galor y Zeira, 1993; Perotti, 1993; Banerjee y Newman, 1993; Piketty, 1997; Aghion y Bolton, 1992).

La ecuación (4) corresponde al Capital Privado per cápita. El signo esperado para los Derechos Políticos es negativo, dado que se intuye que un mayor grado de calidad institucional induce un mayor nivel de inversión privada -y en la escala utilizada 1 indica el máximo grado de libertad posible y 7 el mínimo-. El signo esperado para el Índice de Gobernabilidad es positivo, así como también se espera un signo positivo para la IED, dado que se asume que ésta tiene una contribución neta positiva a la acumulación de capital privado per cápita.

La estimación de la ecuación (3) se realiza, según la metodología descripta, utilizando EGLS y TwoStage EGLS. Se usa como instrumento la variable PIB per cápita en logaritmos retardada dos períodos, lo cual permite obtener estimadores consistentes y más robustos. La Tabla 1 muestra la estimación de la función de crecimiento con convergencia por ambos métodos.

Las estimaciones realizadas corroboran, esencialmente, las hipótesis planteadas. En primer lugar se verifica que todas las variables consideradas inicialmente como potencialmente explicativas son 
estadísticamente significativas. A su vez, los signos observados indican también que las hipótesis planteadas ex ante son correctas.

Se confirma la hipótesis de trabajo de convergencia condicional entre economías, como en Barro y Sala-i-Martin (1990) y Blomström et al. (1994), lo cual sugiere que este fenómeno no sólo ocurre entre países desarrollados y países en vías de desarrollos, como ya señala buena parte de la literatura, sino también entre países en vías de desarrollo entre sí.

Por otro lado, el signo positivo del Índice de Gobernabilidad indica que la mayor Calidad de las Instituciones de los países contribuye al Crecimiento Económico de los mismos, lo cual confirma que los hallazgos para esta región son congruentes con los obtenidos para otras regiones.

También se hallaron que las Políticas de Inversión Pública ejercen un efecto positivo en el Crecimiento, en línea con Bénabou (1996a, 1996b) y Bourguignon y Verdier (2000). Si bien en no se indagó aquí en los medios a través de los cuales tal efecto se materializa, sí se puede destacar que si la Inversión Pública per cápita desplaza parte de la Inversión Privada per cápita, entonces este efecto tiene un menor impacto que otros efectos positivos de la Inversión Pública.

La Inversión Extranjera Directa per cápita promueve también el Crecimiento y este hallazgo confirma la hipótesis de trabajo según la cual esperábamos un impacto positivo de la variable, lo cual es congruente por lo señalado por Ozturk (2007) y gran parte de la literatura de la materia. Se observa, a su vez, que un Modelo de Crecimiento Endógeno es adecuado para la muestra estudiada.

El Capital Humano per cápita es también un determinante en el Crecimiento, tal cual lo esperado, así como lo es la combinación de Inversión Extranjera Directa per cápita y Capital Humano per cápita, lo cual implica que la Inversión Extranjera Directa per cápita tiene un mayor impacto en el Crecimiento Económico cuando el Capital Humano es mayor, consistente con lo hallado por Borensztein et al. (1995), entre otros.

Por otro lado, para probar si los efectos fijos de cada uno de los países estimados pueden o no considerarse iguales, se emplea el test de máxima verosimilitud para la redundancia de los efectos fijos. Se observan p-valores menores que 0,05 , lo cual nos permite afirmar que los efectos fijos de los países deben ser diferentes. El modelo de panel de efectos fijos es, por tanto, más eficiente que aquel de coeficientes constantes. El detalle puede verse en la Tabla 2.

Tabla 1: Estimación de la Ecuación de Crecimiento Económico. Variable dependiente dln $\left(Y_{i t}\right)$.

\begin{tabular}{|c|c|c|}
\hline \multirow[t]{2}{*}{ Variable } & EGLS (Cross-section weights) & $\begin{array}{c}\text { Two-Stage EGLS (Cross- } \\
\text { section weights) }\end{array}$ \\
\hline & Coeficiente; (t-Student) & Coeficiente; (t-Student) \\
\hline C & 1,$64992 ;(-8,76158)$ & 2,$78152 ;(-7,73298)$ \\
\hline $\operatorname{Ln}\left(Y_{i t-1}\right)$ & $-0,1923 ;(-9,2617)$ & $-0,3241 ;(-7,8364)$ \\
\hline $\mathrm{dLn}\left(\mathrm{KHU}_{\mathrm{it}}\right)$ & 0,$03242 ;(-1,56848)$ & 0,$04073 ;(-3,70268)$ \\
\hline $\mathrm{GOB}_{\mathrm{it}-1}$ & 0,$02057 ;(-2,18123)$ & 0,$02974 ;(-2,98652)$ \\
\hline $\mathrm{dLn}\left(\mathrm{PuK}_{\mathrm{it}}\right)$ & 0,$02689 ;(-2,32231)$ & 0,$01818 ;(-2,86144)$ \\
\hline $\mathrm{dLn}\left(\operatorname{PrK}_{\mathrm{it}}\right)$ & 0,$06645 ;(-7,45855)$ & 0,$05013 ;(-9,28846)$ \\
\hline $\mathrm{dLn}\left(\mathrm{IED}_{\text {it }}\right)$ & 0,$00399 ;(-1,38856)$ & 0,$0056 ;(-2,14155)$ \\
\hline $\mathrm{dLn}\left(\mathrm{IED}_{\mathrm{it}-1}{ }^{*} \mathrm{KHU}_{\mathrm{it}-1}\right)$ & 0,$00779 ;(-3,46289)$ & 0,$00907 ;(-4,01544)$ \\
\hline $\mathrm{R}^{2}$ (ponderado) & 0,743726 & 0,781825 \\
\hline $\mathrm{R}^{2}$-ajustado (ponderado) & 0,66364 & 0,713645 \\
\hline Durbin-Watson (ponderado) & 1,802825 & 1,7571 \\
\hline $\mathrm{R}^{2}$ (no ponderado) & 0,601462 & 0,574627 \\
\hline
\end{tabular}


Tabla 2: Test de Máxima verosimilitud para la redundancia de los coeficientes de Efectos Fijos de la ecuación de Crecimiento Económico con variable dependiente $\operatorname{dln}\left(\mathrm{Y}_{\mathrm{it}}\right)$ frente a la ecuación con coeficientes constantes.

\begin{tabular}{|l|c|c|c|}
\hline Effects Test & Estadístico & d.f. & Prob. \\
\hline Cross-section F & 2,741931 & $(13,64)$ & 0,0038 \\
\hline
\end{tabular}

Por último, se analizó la relación entre la Inversión Extranjera Directa per cápita y la Inversión Privada per cápita con la estimación de la ecuación (4). Los resultados se presentan en la Tabla 3.

Esta ecuación indica que la Inversión Extranjera Directa per cápita es significativa en la determinación de la acumulación de Capital Privado per Cápita. Dado que su impacto neto es positivo, al igual que en Borensztein et al., 1995, podemos inferir que la Inversión Extranjera Directa contribuye a la Formación de Capital en términos per cápita y, aun cuando podría asumirse que compite con la Inversión Doméstica, debe reconocerse un efecto neto es positivo. Posiblemente en las economías estudiadas existen más oportunidades rentables que aquellas que pueden ser financiadas por los agentes privados internos, con lo que la apertura del mercado de capitales pasa a ser un factor importante en el Crecimiento Económico.

A su vez, se observa que el nivel de Capital Humano también determina la formación de Capital Privado per Cápita, confirmando lo señalado por otros autores e indicado anteriormente.

El mayor grado de calidad institucional promueve también la acumulación de Capital. Esto es observable tanto en el signo positivo de la variable de Gobernabilidad como en el negativo de Derechos Políticos. Estos resultados sugieren que la protección de los derechos individuales y de propiedad privada y el desarrollo de las Instituciones destinadas a asegurar el imperio de la ley impulsan una mayor acumulación de Capital Privado per cápita.

Tabla 3: Estimación de la Ecuación de Inversión Privada. Variable dependiente $\ln \left(\operatorname{PrK}_{\mathrm{it}}\right)$.

\begin{tabular}{|c|c|c|c|c|}
\hline Variable & Coeficiente & Std. Error & t-Statistic & Prob. \\
\hline C & 6,391026 & 0,185137 & 34,52049 & 0 \\
\hline $\operatorname{Ln}\left(\operatorname{IED}_{i t}{ }^{*} K H U_{i t}\right)$ & 0,037639 & 0,017417 & 2,161051 & 0,0336 \\
\hline $\operatorname{Ln}\left(\operatorname{IED}_{\mathrm{it}-1}\right)$ & 0,045862 & 0,013998 & 3,276442 & 0,0015 \\
\hline$D P_{i t-1}$ & $-0,069469$ & 0,014794 & $-4,695644$ & 0 \\
\hline $\mathrm{GOB}_{\text {it }}$ & 0,296956 & 0,071809 & 4,135334 & 0,0001 \\
\hline \multicolumn{5}{|c|}{ Especificación de Efectos } \\
\hline \multicolumn{5}{|c|}{ Cross-section fixed (dummy variables) } \\
\hline $\mathrm{R}^{2}$ (ponderado) & 0,999755 & \multicolumn{2}{|c|}{$\mathrm{R}^{2}$ ajustado (ponderado) } & 0,999703 \\
\hline $\mathrm{R}^{2}$ (no ponderado) & 0,896907 & \multicolumn{2}{|c|}{ Durbin-Watson (ponderado) } & 1,292257 \\
\hline
\end{tabular}

\section{CONCLUSIONES}

En este trabajo se analizó el impacto de la Inversión Extranjera Directa en el Crecimiento Económico de 14 países latinoamericanos a través de su relación con la evolución del PIB per cápita y el Capital Privado per cápita. Al respecto se halló que la Inversión Extranjera Directa impulsa tanto la Formación de Capital como el Crecimiento Económico, todo medido en términos per cápita y confirmamos que un Modelo de Crecimiento Endógeno es adecuado para la explicación del fenómeno en la muestra analizada. 
Los resultados sugieren que la Calidad Institucional es un determinante del Crecimiento. La protección de los derechos de propiedad impulsan la inversión y con esto el crecimiento y, a un mayor grado de libertad en el país, corresponde una mayor acumulación de Capital Privado per cápita.

También se observó convergencia condicional en los países estudiados en términos de Ingreso per cápita, algo que se esperaba desde el planteo de las ecuaciones y que, además, está en concordancia con lo establecido por una buena parte de la literatura.

En el presente estudio se halló que la Inversión Pública promueve el Crecimiento Económico, lo cual podría explicarse a través de mejoras producidas en estructuras productivas y regulatorias de las economías.

Por último, el Capital Humano promueve tanto la acumulación del Capital Privado como el Crecimiento Económico, lo cual es un dato importante para aquellos organismos haciendo las políticas públicas y concordante con la mayoría de la literatura al respecto.

\section{REFERENCIAS}

Aghion, P. y P. Bolton; Distribution and growth in models of imperfect capital markets, European Economic Review: 36, 603-611 (1992).

Alesina, A. y D. Rodrick; Distributive politics and economic growth, Quarterly Journal of Economics: 436, 465-490 (1994).

Alfaro, L., A. Chanda, S. Kalemli-Ozcan y S. Sayek; FDI and economic growth: the role of local financial markets, Journal of International Economics: 64(1), 89-112 (2004).

Balasubramanyam, V., M. Salisu, y D. Sapsford; Foreign Direct Investment and Growth in EP and IS Countries, The Economic Journal: 106(434), 92-105 (1996).

Banerjee, A. y A. Newman; Occupational choice and the process of development, Journal of Political Economy: 101, 274-298 (1993).

Barro, R y X. Sala-i-Martin; Economic Growth and Convergence Across the United States NBER Working Paper No. W3419 (1990).

Bénabou, R.; Unequal societies, NBER Working Paper 5583 (1996a).

Bénabou, R.; Inequality and growth, NBER Macroeconomic Annual 1996, MIT Press, 11-74, Cambridge, MA., Estados Unidos (1996b).

Bertola, G.; Market structure and income distribution in endogenous growth models, American Economic Review: 83, 1184-1199 (1993).

Blomström, M., R. Lipsey, y M. Zejan; What Explains Developing Country Growth?, In Convergence and Productivity: Gross-National Studies and Historical Evidence by Baumol, W., Nelson, R. y E. Wolff, Oxford University Press, Oxford (1994).

Bond, Stephen R., Hoeffler, Anke y Temple, Jonathan R.W., GMM Estimation of Empirical Growth Models, CEPR Discussion Paper No. 3048 (2001). SSRN: http://ssrn.com/abstract=290522. Acceso: Septiembre de 2007.

Borensztein, E., J. de Gregorio y J-W. Lee; How Does Foreign Direct Investment Affect Economic Growth?, NBER Working: Paper No. W5057 (1995).

Bourguignon, F. y T. Verdier, Oligarchy, democracy, inequality, and growth, Journal of Development Economics: 62, 285-313 (2000). 
Boyd, J. y B. Smith; Intermediation and the Equilibrium Allocation of Investment Capital: Implications for Economic Development, Journal of Monetary Economics: 30, 409-432 (1992).

Carkovic, M.V. y R. Levine; Does Foreign Direct Investment Accelerate Economic Growth? en Moren, T. H., E. M. Grahma y M. Blomström (Eds.), Does Foreign Direct Investment Promote Development?, Institute for International Economics, 195-220, Washington, WA., Estados Unidos (2005).

Caselli, F., G. Esquivel y F. Lefort; Reopening the convergence debate: A new look at cross-country growth empirics, Journal of Economic Growth. Springer Netherlands: 1(3), 363-389 (1996).

De Mello, L.R.; Foreign Direct Investment-Led Growth: Evidence from Time series and Panel Data, Oxford Economic Papers: 51 (1999).

Galindo, M.A. y L. Escot; International Capital Flows, Convergence and Growth, The Journal of Economic Asymmetries: 1(1), 49-69 (2004).

Galor, O. y J. Zeira; Income distribution and macroeconomics, Review of Economic Studies: 60(1), 35-52 (1993).

Hermes, N. y R. Lensink; Foreign Direct Investment, Financial Development and Economic Growth, SOM Theme E Workings Papers: 27 (2000).

Kaufmann, D., A. Kraay y M. Mastruzzi; Governance Matters IV: Governance Indicators for 19962004, The World Bank, mimeo (2005).

Lipsey, R.E.; The Role of Foreign Direct Investment in International Capital Flows, NBER Working Paper n. 7094 (2000).

Ozturk, I.; Foreign Direct Investment - Growth Nexus: A Review of the Recent Literature, International Journal of Applied Econometrics and Quantitative Studies: 4(2), 79-98 (2007).

Perotti, R.; Political equilibrium, income distribution and growth, Review of Economic Studies: 60, 755-776 (1993).

Persson, T. y G. Tabellini; Is inequality harmful for growth?, American Economic Review: 84, 600-621 (1994).

Piketty, T.; The dynamics of wealth distribution and the interest rate with credit rationing, Review of Economic Studies: 64(2), 173-189 (1997).

Romer, P.; Idea Gaps and Object Gaps in Economic Development, Journal of Monetary Economics: 32(3), 543-573 (1993). 